\title{
Lowering cholesterol in statin-intolerant patients
}

Patients with dyslipidemia who cannot tolerate statin therapy could benefit from supplementation with red yeast rice, report David Becker and colleagues from the University of Pennsylvania, USA.

Compared with the placebo group (who took dummy supplements), "the group that took red yeast rice had significant drops in LDL cholesterol and total cholesterol," comments Becker.

Statins are highly effective cholesterollowering drugs that can prevent coronary artery disease and are, on the whole, well tolerated. A small percentage of patients do, however, experience severe adverse effects. "The most common problem is the development of muscle pain or

fatigue ... called statin-associated myalgia," Becker explains. Co-researcher Ram Gordon comments that, although studies of some statins suggest as few as $2 \%$ of patients develop muscle aches, “...most doctors have found in their actual clinical practice that up to $15 \%$ of people have these muscle problems, and have to stop their medication." These patients still require therapy for hyperlipidemia, and may seek alternative treatments. Becker had previously noted that some of his patients assigned to statin therapy had switched to red yeast rice-a herbal supplement that contains low doses of

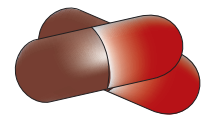
naturally occurring lovastatin and reduces LDL-cholesterol levels. These patients had achieved acceptable cholesterol levels without the adverse effects associated with statins. Acting on this anecdotal evidence, Becker's team sought to study the effects of red yeast rice supplementation in a randomized, controlled trial.

\section{4 ...the group that took red yeast rice had significant drops in LDL cholesterol and total cholesterol 77}

The investigators enrolled 62 patients with high cholesterol who had stopped taking statins after developing statinassociated myalgia. All patients were enrolled in a 12-week therapeutic lifestyle change program at the same time as being randomly assigned to receive either three $600 \mathrm{mg}$ capsules of red yeast rice or three placebo capsules (identical in colour, size and shape) twice daily for 24 weeks.

At week 12 both LDL-cholesterol and total-cholesterol levels were markedly lower in patients taking the supplements than in those taking placebo. By week 24 , $30 \%$ of patients in the red yeast rice group achieved an LDL-cholesterol level of 100 $\mathrm{mg} / \mathrm{dl}$ or lower, compared with $6.9 \%$ of those in the placebo group. There was no prominent difference in the development of myalgias between the groups. Becker notes that this result was surprising, as patients with a history of statinassociated myalgia are prone to relapse of symptoms following treatment with another statin.

"There is a problem with red yeast rice, as there is with many nutraceuticals, because they are not regulated by the FDA," Becker warns. The observed effective reduction in levels of LDL cholesterol and total cholesterol by red yeast rice is, however, a promising result that warrants further study in a larger, longer, multicenter trial, the authors conclude.

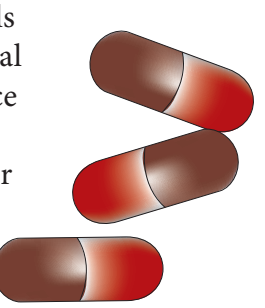

Lisa Richards

Original article Becker, D. J. et al. Red yeast rice for dyslipidemia in statin-intolerant patients: a randomized trial. Ann. Intern. Med. 150, 830-839 (2009). 